\title{
Predictive factors and prognostic value of pathologic complete response of ipsilateral supraclavicular lymph nodes in breast cancer after neoadjuvant chemotherapy
}

\author{
Jiujun Zhu ${ }^{1}$, Dechuang Jiao ${ }^{1}$, Xuhui Guo ${ }^{1}$, Jianghua Qiao ${ }^{1}$, Youzhao Ma ${ }^{1}$, Jingyang Zhang ${ }^{1}$, Hui Chen ${ }^{2}$, \\ Hui Xiao ${ }^{1}$, Yue Yang ${ }^{1}$, Zhenduo Lu ${ }^{1}$, Zhenzhen Liu ${ }^{1}$ \\ ${ }^{1}$ Department of Breast Surgery, Affiliated Cancer Hospital of Zhengzhou University, Henan Cancer Hospital, Zhengzhou 450008, China; ${ }^{2}$ People's \\ Hospital of Zhengzhou, Zhengzhou 450008, China \\ Contributions: (I) Conception and design: Z Lu, Z Liu; (II) Administrative support: J Zhu; (III) Collection and assembly of data: J Zhu, D Jiao, X Guo, \\ J Qiao, Y Ma, J Zhang, H Chen, H Xiao, Y Yang; (IV) Data analysis and interpretation: J Zhu, D Jiao; (V) Manuscript writing: All authors; (VI) Final \\ approval of manuscript: All authors. \\ Correspondence to: Zhenzhen Liu. Department of Breast Surgery, Affiliated Cancer Hospital of Zhengzhou University, Henan Cancer Hospital, \\ Zhengzhou 450008, China. Email: liuzhenzhen73@126.com.
}

Background: Breast cancer with ipsilateral supraclavicular lymph node metastasis is one of the indicators of poor prognosis. Patients who attain pathologic complete response in breast and axillary sites have improved survival and are highest in aggressive HR-HER2- and HER2-positive tumor subtypes. However, there is no study on the related factors and prognostic value of supraclavicular pathologic complete response in breast cancer after neoadjuvant chemotherapy. The aim of our work was to investigate the factors and prognostic significance of pathologic complete response of ipsilateral supraclavicular lymph node metastasis in breast cancer after neoadjuvant chemotherapy.

Methods: A total of 214 patients with breast cancer who had primary ISLN metastasis, receiving NAC and subsequent ISLN dissection, were retrospectively and consecutively reviewed. Univariate and multivariate analyses were performed using $\chi^{2}$ test and the logistic regression model, and the prognosis was analyzed by Kaplan-Meier curve.

Results: All patients included were women who were 26-74 years old. The rate of supraclavicular pathologic complete response (pCR) was 53.7\%. Multivariate analysis showed that the expression of Ki67, breast pCR, and axillary pCR were independent predictors of supraclavicular pCR $(\mathrm{P}<0.05)$. After a median follow-up of 16.2 months, the risk of recurrence and metastasis in patients with supraclavicular pCR was half reduced compared to that of the non-pCR group (HR 0.51, 95\% CI, 0.32-0.80, P<0.01), mainly manifested in HR-HER2- and HER2-positive disease.

Conclusions: The expression level of Ki67, breast pCR, and axillary pCR were independent predictors of supraclavicular pCR. Supraclavicular pCR was an independent predictor of disease-free survival (DFS). Surgical removal of supraclavicular lymph nodes can accurately evaluate the rate of supraclavicular pCR, which is of great significance for patient prognosis.

Keywords: Breast neoplasms; neoadjuvant chemotherapy; supraclavicular lymph node metastasis; pathologic complete response (pCR); prognosis

Submitted Jun 22, 2019. Accepted for publication Sep 24, 2019.

doi: $10.21037 /$ atm.2019.10.22

View this article at: http://dx.doi.org/10.21037/atm.2019.10.22 


\section{Introduction}

Breast cancer with ipsilateral supraclavicular lymph node metastasis (ISLNM) is one of the indicators of poor prognosis (1). In 2003, patients with ISLNM were included in the stage IIIc category of the tumor-nodemetastasis classification from M1 stage in the sixth edition of the American Cancer Commission staging system (AJCC-TNM) (2). It is suggested that this kind of patient be reclassified from advanced incurable individuals to locally advanced individuals with the possibility of cure. The treatment recommendation was also changed into a comprehensive scheme of chemotherapy plus surgery followed by radiotherapy and endocrine therapy.

Only a few retrospective reports have focused on the prognosis of patients with synchronous ISLN metastasis; however, these studies are important because the prospective study of these patients is unattainable. These studies also have several limitations including very small sample sizes, inhomogeneous study populations, selection bias, and absence of pathological proof for ISLN metastasis, which results in over staging.

Neoadjuvant chemotherapy (NAC) combined with or without targeted therapy is already the standard treatment model for locally advanced breast cancer. Patients who attain pathologic complete response (pCR) in breast and axillary sites have improved survival and are highest in aggressive HR-HER2 - and HER2-positive tumor subtypes (3). However, there is no study on the related factors and prognostic value of supraclavicular pCR in breast cancer after NAC. We aimed to investigate the influencing factors of supraclavicular pCR and its relationship with disease-free survival (DFS) for breast cancer patients with pathologically proven synchronous ISLN metastases.

\section{Methods}

\section{Study population}

We retrospectively and consecutively identified a total of 214 patients treated with NAC followed by breast, axillary, and supraclavicular lymph nodal surgery in the Department of Breast Surgery, Henan Cancer Hospital from August 1, 2012 to April 10, 2018. The key inclusion criteria were as follows: (I) the breast lesions were diagnosed as primary unilateral invasive breast cancer by coarse needle biopsy before chemotherapy; (II) the ipsilateral supraclavicular lymph nodes (ISLN) were confirmed to be cancer metastasis by fine needle or coarse needle puncture; (III) estrogen receptor (ER), progesterone receptor (PR), human epidermal growth factor receptor 2 (HER2), and Ki-67 data integrity; (IV) acceptable NAC $\geq 2$ cycles. The exclusion criteria were as follows: (I) bilateral breast cancer; (II) distant metastasis at diagnosis; (III) simultaneous or previous history of other malignant tumors. The chemotherapy regimen contained anthracycline only in $33(15.5 \%)$ patients and taxane only in $25(11.7 \%)$ patients. A total of $155(72.8 \%)$ patients received both anthracycline and taxane as NAC. Meanwhile, only $27.8 \%(\mathrm{n}=30)$ of patients used trastuzumab in the HER2-positive disease $(n=108)$. After NAC, all individuals received standard modified or breast-conserving therapy and ISLN dissection followed by radiotherapy. Concurrent adjuvant endocrine therapy for hormone receptor (HR)-positive disease was recommended. Outpatient registration and telephone follow-up continued until January 26, 2019, with the median length of follow-up being 16.2 months (range, 2.1-74.1 months), and 9 patients being lost to follow-up (4.2\%).

\section{Outcomes}

Pathologic specimens were reviewed by dedicated breast pathologists at Henan Cancer Hospital. We performed immunohistochemical (IHC) staining for ER, PR, HER2, Ki-67, androgen receptor (AR), cytokeratin (CK5/6), and epidermal growth factor receptor (EGFR) on 5 -mm paraffin sections. The criteria were as follows $(3,4)$ : positive ER or PR ( $>1 \%$ of tumor cell nuclei staining) or positive $\mathrm{AR}(>10 \%$ of tumor cell nuclei staining) were collapsed into a single HR or AR "positive" category. HER2 positive was defined as 3 + receptor overexpression on IHC staining and/ or gene amplification on fluorescence in situ hybridization. "Low expression" was defined as Ki-67 levels below 30\%, and "high expression" as levels above $30 \%$. Cases were considered as EGFR positive or CK5/6 positive if any membranous and/or cytoplasmic staining was detected in the tumor cells, even if focal. Breast pCR was defined as no evidence of residual invasive and in situ carcinoma (ypT0). Axillary pCR or supraclavicular pCR was defined as no evidence of metastatic carcinoma (ypN0). DFS was defined as invasive ipsilateral tumor recurrence, invasive contralateral breast cancer, local or regional invasive recurrence, and distant recurrence from time of operation.

\section{Statistical analysis}

All statistical analyses were performed using Graphpad 
Prism 5.0, SPSS statistical software (version 23, SPSS Inc.) and $\mathrm{R}$ (version 3.4.3). A 2 -sided $\mathrm{P}<0.05$ was considered to be statistically significant. Categorical variables were compared using a 2 -sided $\chi^{2}$ test. Binary logistic regression analysis was used for multivariate analysis. Kaplan-Meier estimates of time-to-event end points were calculated with the use of log-rank tests. We used Cox regression models to estimate hazard ratios (HR) and $95 \%$ confidence intervals (95\% CI). The prognostic performance of breast pCR, axillary pCR, and supraclavicular pCR was evaluated using $\mathrm{P}$ value, concordance index (C-index), and integrated area under the (time-dependent receiver operating characteristic) curve (AUC).

\section{Results}

We enrolled 214 female patients, aged 26 to 74 years (median 51.5 years), and $51.9 \%(n=111)$ of the women were diagnosed while still premenopausal. Findings from this study showed that $15.9 \%(\mathrm{n}=33)$ of patients were diagnosed at stage cT3 and $18.8 \%(n=39)$ at stage cT4 disease. Based on immunostaining data for the three markers used (ER, PR, HER2), 59 invasive tumors were classified as HR+HER2- (28.1\%), 51 were HR+HER2+ (24.3\%), 57 were HR-HER2+ (27.1\%), and 43 were HRHER2- (20.5\%). Of the post-operation patients, 70 (32.7\%) patients achieved pCR in the breast, $69(32.2 \%)$ in the axilla, and $113(53.7 \%)$ in the supraclavicular area. There were 32 recurrence events in the supraclavicular pCR group (28.3\%) compared with 45 in the non-supraclavicular pCR group $(58.4 \%)$ (Table 1$)$.

The proportion of supraclavicular pCR was $53.7 \%$ after NAC. Univariate analysis showed that it was correlated with the expression level of Ki67, chemotherapy regimen, number of chemotherapy cycles, the rate of axillary pCR, and rate of breast $\mathrm{pCR}(\mathrm{P}<0.05)$. Meanwhile, the correlation between age, menstrual status, tumor location, $\mathrm{T}$ stage, HR status, HER2 status, AR, EGFR, CK5/6 and molecular subtypes, and supraclavicular pCR were not statistically compelling. Multivariate analysis demonstrated that the expression level of Ki67, axillary pCR, and breast pCR were independent predictors of supraclavicular pCR $(\mathrm{P}<0.05)$. The patients with a high expression of Ki67, breast pCR and axillary pCR were increasingly likely to obtain supraclavicular pCR (Table 1).

Of the patients with breast pCR, $93.0 \%$ of patients achieved a supraclavicular pCR in the axillary pCR group, compared with $70.4 \%$ in the non-axillary pCR group
$(\mathrm{P}=0.01)$. It was to be expected that among the patients with non-breast $\mathrm{pCR}$, there was a decreased probability of obtaining a supraclavicular pCR, which appeared in $65.4 \%$ of these patients in the axillary pCR group, compared with $33.1 \%$ in the non-axillary pCR group $(\mathrm{P}<0.01)$. The supraclavicular pCR rate was $33.1 \%$ in those patients who did not have PCR in the breast or axilla (Table 2). The highest rate of supraclavicular pCR was obtained in patients with both breast and axillary pCR and a high expression of Ki67 (95.0\%, 38 of 40).

At the cutoff date (January 26, 2019), the median duration of follow up was 16.2 months. In the cohort of patients with supraclavicular pCR, there were significantly fewer DFS events compared to those patients in the nonsupraclavicular pCR group (32 vs. 45 events). The DFS was $71.7 \%$ in the supraclavicular pCR group and $54.1 \%$ in the non-supraclavicular pCR group (HR 0.51, 95\% CI, 0.320.80, $\mathrm{P}<0.01)$. PCR also predicted a better DFS survival in patients who had obtained a pCR in all of the breast, axillary, and supraclavicular (tpCR) sites when compared with those with one or more of the three sites without $\mathrm{pCR}$ (non-tpCR) ( $\mathrm{P}=0.01)$ (Figure 1). Prognosis according to intrinsic subtype was analyzed separately for patients with or without supraclavicular pCR. DFS was notably increased only in highly aggressive HR-HER2 - and HER2+ tumors $(\mathrm{P}<0.05)$ (Figures 2,3).

Findings from additional exploratory subgroup analyses by single COX regression revealed that the prognostic effect of supraclavicular pCR for DFS was homogeneous among different patient subgroups. Generally, the effect was more pronounced in patients with HR negative, HER2 positive, high expression of Ki67, AR positive, CK5/6 positive, EGFR positive, ER-HER2-, or received anthracycline combined taxane-based chemotherapy tumors when they had a supraclavicular pCR $(\mathrm{P}<0.05)$ (Figure 3).

To adjust for confounding factors, a Cox regression model was performed to test effects of supraclavicular pCR interactions with DFS. The HR for DFS favoured those patients achieving supraclavicular pCR after neoadjuvant therapy (HR 0.46, 95\% CI, 0.26-0.84, $\mathrm{P}=0.01$ ), which demonstrated that supraclavicular pCR was an independent and strong predictor for DFS. Unexpectedly, there was no such relationship with breast pCR and axillary pCR (Table 3).

The iAUC of the time-dependent ROC curve from 6 to 40 months was calculated as a measurement of discrimination. Integrated AUC was calculated by averaging all AUC values. A larger iAUC indicates a better predictability of survival. With regard to prognostic 
Table 1 Baseline patient characteristics and corresponding sPCR rates $(\mathrm{N}=214)$

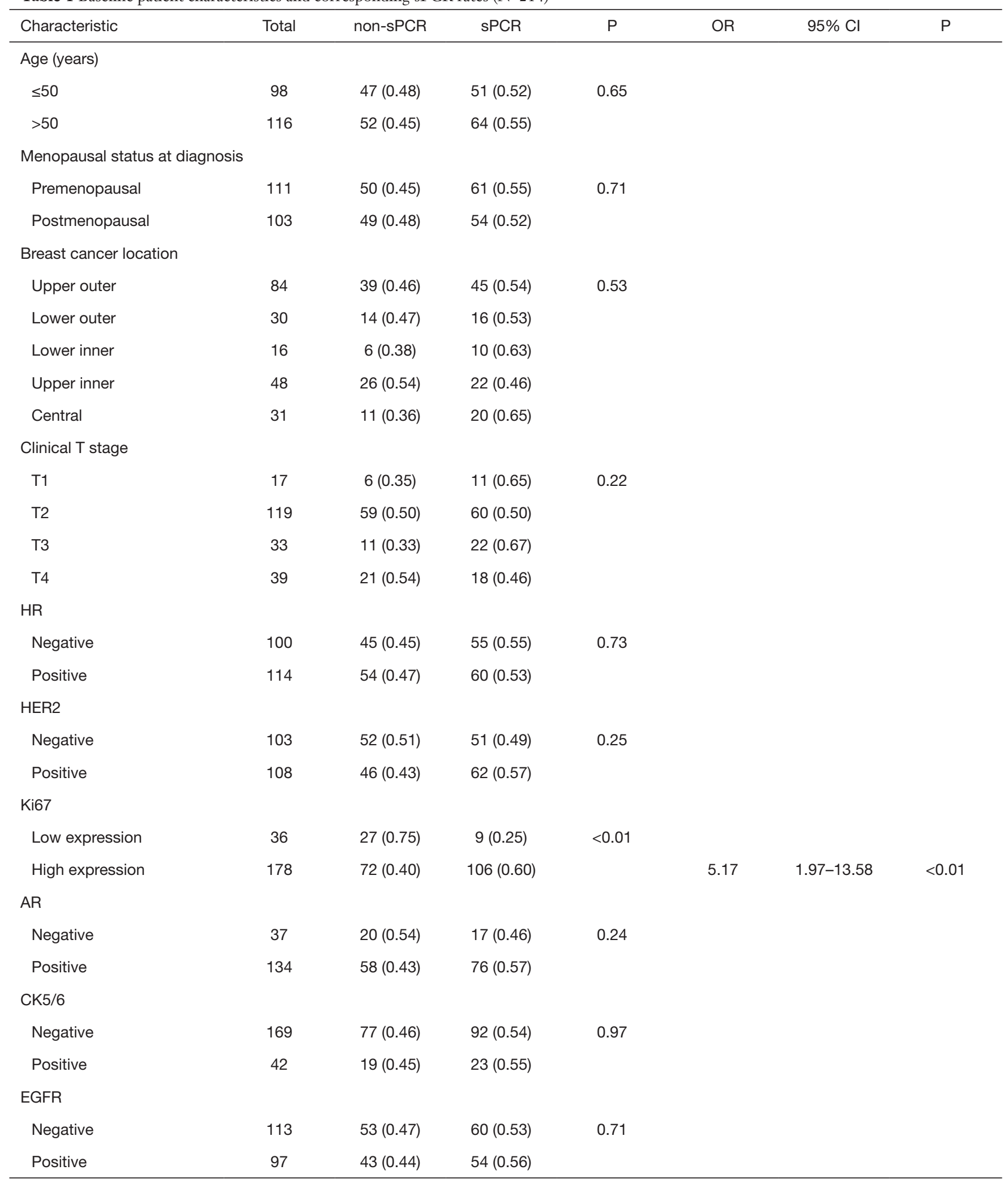

Table 1 (continued) 
Table 1 (continued)

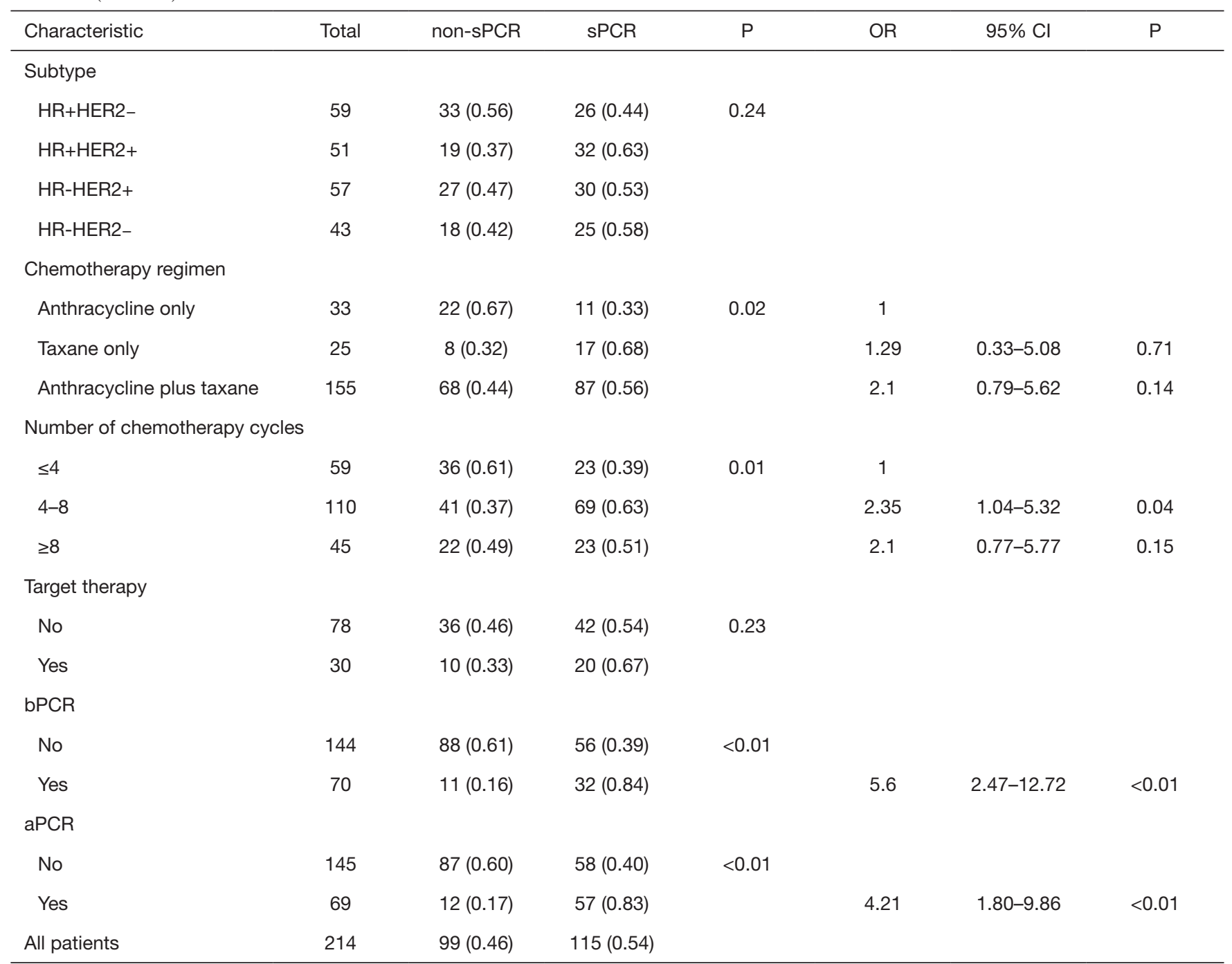

HR, hormone receptor; HER2, human epidermal growth factor receptor 2; AR, androgen receptor; CK5/6, cytokeratin; EGFR, epidermal growth factor receptor; PCR, pathologic complete response; sPCR, supraclavicular PCR; bPCR, breast PCR; aPCR, axillary PCR; -, negative; +, positive.

Table 2 Correlation between bPCR, aPCR, and sPCR

\begin{tabular}{|c|c|c|c|c|}
\hline bPCR & aPCR & \multicolumn{2}{|c|}{ sPCR } & $P$ \\
\hline \multirow[t]{2}{*}{ No } & No & $79(0.67)$ & $39(0.33)$ & $<0.01$ \\
\hline & Yes & $9(0.35)$ & $17(0.65)$ & \\
\hline Yes & No & $8(0.30)$ & $19(0.70)$ & 0.01 \\
\hline
\end{tabular}

PCR, pathologic complete response; sPCR, supraclavicular PCR; bPCR, breast PCR; aPCR, axillary PCR. 


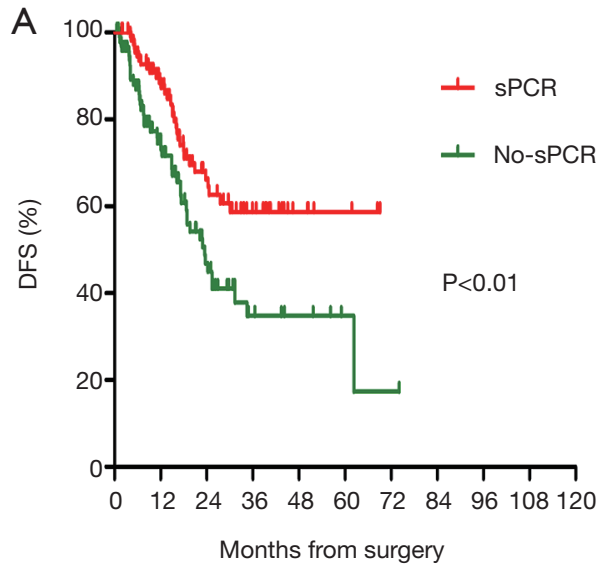

B

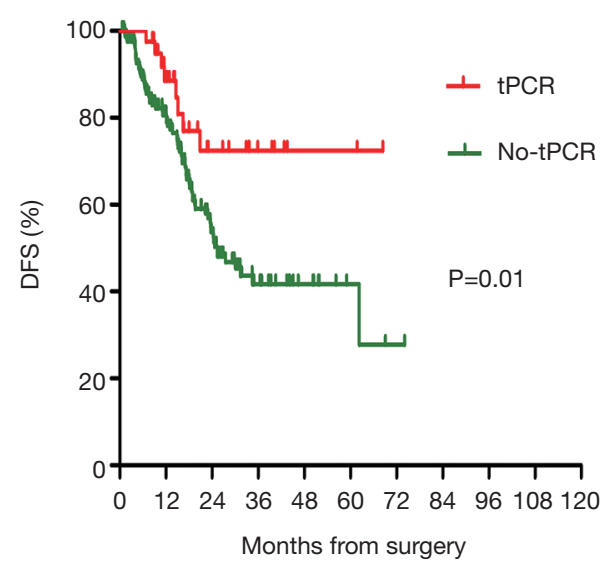

Figure 1 Prognostic impact of sPCR (A) and tPCR (B) on disease-free survival (DFS). The DFS was 71.7\% in the supraclavicular pCR group and $54.1 \%$ in the non-supraclavicular pCR group. PCR also predicted a better DFS in patients who had obtained a pCR in all of the breast axillary and supraclavicular (tpCR) sites when compared with those with one or more of the three sites without pCR (non-tpCR).

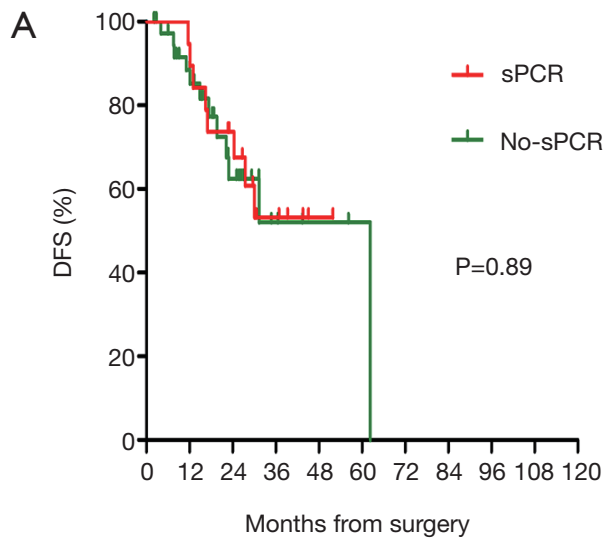

B

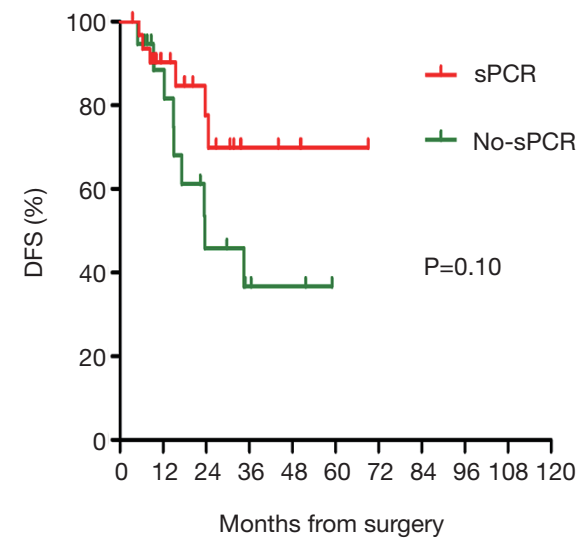

C

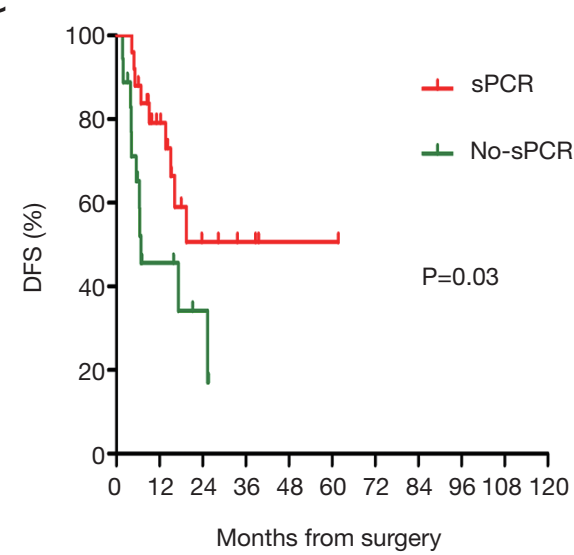

$\mathrm{D}$

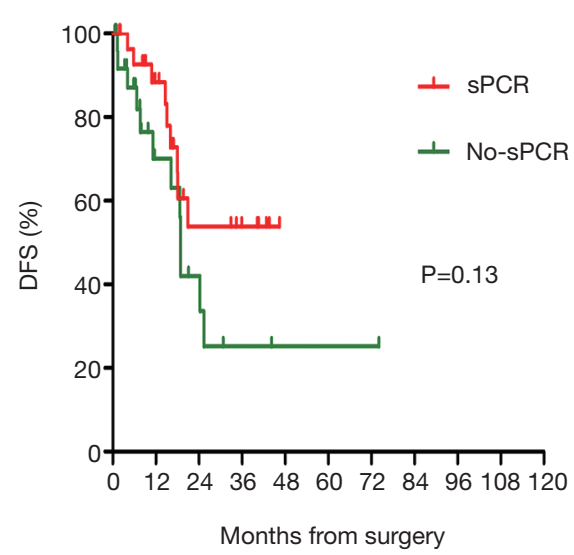

Figure 2 Prognostic impact of sPCR on disease-free survival (DFS) according to breast cancer intrinsic subtype. DFS was notably increased only in highly aggressive HR-HER2- and HER2+ tumors. (A) HR+HER2-, (B) HR+HER2+, (C) HR-HER2-, (D) HR-HER2+. 


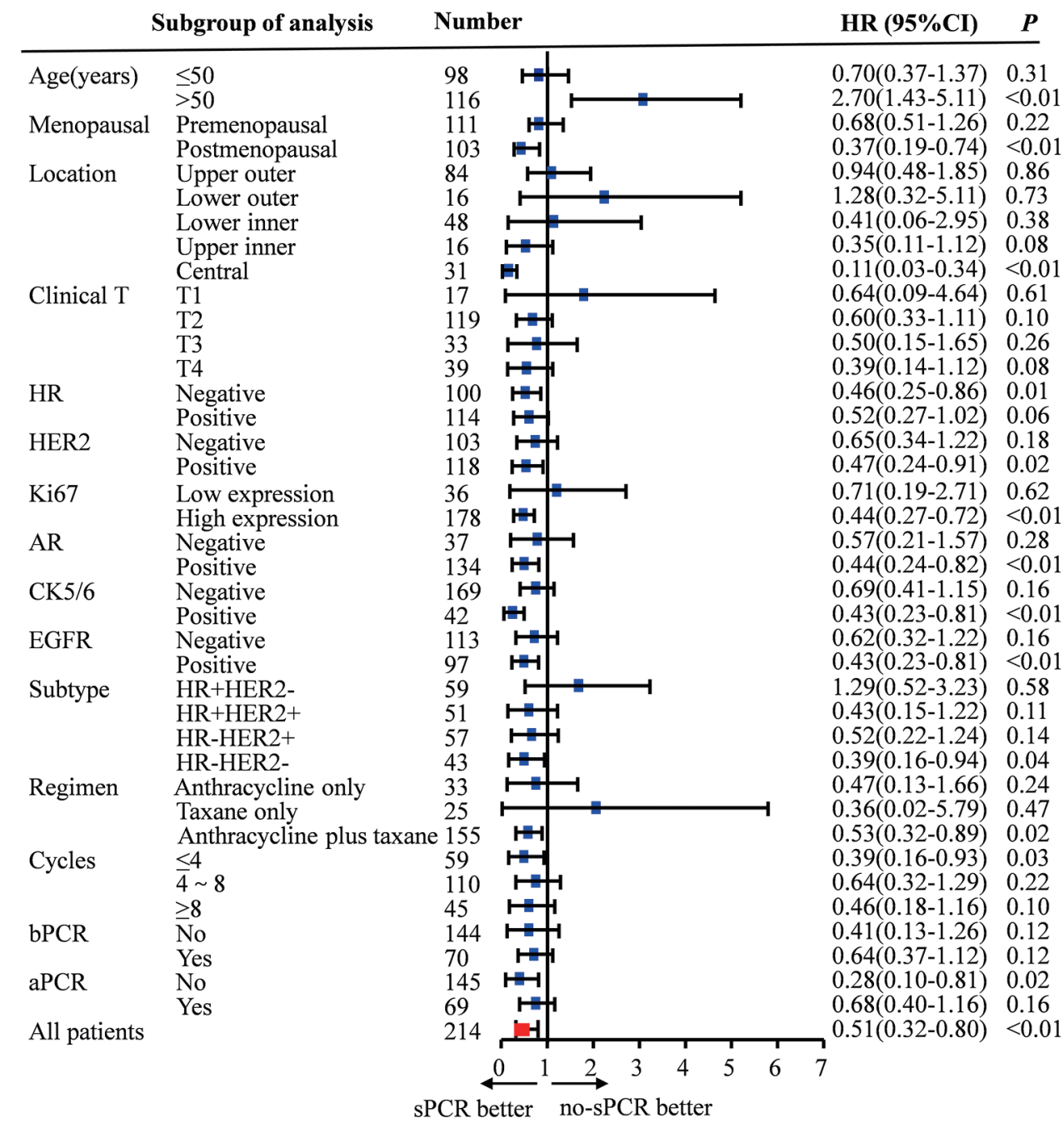

Figure 3 Subgroup analysis of sPCR on disease-free survival (DFS). Exploratory subgroup analyses by single COX regression revealed that the prognostic effect of supraclavicular pCR for DFS was homogeneous among different patient subgroups.

ability, supraclavicular pCR performed better than the commonly used breast pCR and axillary pCR (Figure 4). The AUC of supraclavicular pCR was the highest between 6 and 40 months, followed by breast pCR and axillary pCR. The iAUC of supraclavicular pCR was 0.63 , whereas those of breast pCR and axillary pCR were 0.55 and 0.54 , respectively. Although there was no statistical significance, the C-index of supraclavicular pCR $(0.69,95 \%$ CI, $0.58-$ $0.82)$ was also much higher than that of breast pCR $(0.63$, $95 \%$ CI, $0.48-0.78, \mathrm{P}=0.20)$ and axillary pCR $(0.60,95 \%$ $\mathrm{CI}, 0.44-0.77, \mathrm{P}=0.14)$, confirming the strong prognostic power of supraclavicular pCR.

\section{Discussion}

The supraclavicular lymph node is one of the lymphatic drainage fields from the primary tumor to the draining lymph nodes, while the likelihood of lymphatic drainage from the unilateral breast to the ipsilateral supraclavicular is $3.1 \%(5)$. Although the incidence of isolated ISLNM in breast cancer is approximately only $1-4 \%$ of newly diagnosed cases, it is one of the more important indicators for poor prognosis (6). The occurrence of ISLM in breast cancer has been significantly correlated with a variety of clinicopathological factors. Consistent with previous 
Table 3 Univariate and multivariate analysis of COX regression

\begin{tabular}{|c|c|c|c|c|c|}
\hline Characteristic & Total & $\mathrm{P}$ & $\mathrm{HR}$ & $95 \% \mathrm{Cl}$ & $\mathrm{P}$ \\
\hline$\leq 50$ & 98 & & & & \\
\hline$>50$ & 116 & 0.44 & & & \\
\hline \multicolumn{6}{|c|}{ Menopausal status at diagnosis } \\
\hline Postmenopausal & 103 & 0.68 & & & \\
\hline \multicolumn{6}{|c|}{ Breast cancer location } \\
\hline Upper outer & 84 & & & & \\
\hline Lower outer & 30 & & & & \\
\hline Central & 31 & 0.58 & & & \\
\hline \multicolumn{6}{|l|}{ Clinical T stage } \\
\hline $\mathrm{T} 1$ & 17 & & & & \\
\hline $\mathrm{T} 2$ & 119 & & & & \\
\hline T3 & 33 & & & & \\
\hline $\mathrm{T} 4$ & 39 & 0.48 & & & \\
\hline \multicolumn{6}{|l|}{ HR } \\
\hline Negative & 100 & & & & \\
\hline \multicolumn{6}{|l|}{ Ki67 } \\
\hline Low expression & 36 & & & & \\
\hline High expression & 178 & 0.57 & & & \\
\hline \multicolumn{6}{|l|}{$A R$} \\
\hline Negative & 37 & & & & \\
\hline Positive & 134 & 0.02 & 1.56 & $0.67-3.53$ & 0.29 \\
\hline \multicolumn{6}{|l|}{ CK5/6 } \\
\hline Negative & 169 & & & & \\
\hline Positive & 42 & 0.03 & 2.39 & $1.02-5.60$ & 0.04 \\
\hline \multicolumn{6}{|l|}{ EGFR } \\
\hline Negative & 113 & & & & \\
\hline Positive & 97 & 0.04 & 1.44 & $0.69-3.01$ & 0.33 \\
\hline
\end{tabular}

Table 3 (continued) 
Table 3 (continued)

\begin{tabular}{|c|c|c|c|c|c|}
\hline Characteristic & Total & $\mathrm{P}$ & $\mathrm{HR}$ & $95 \% \mathrm{Cl}$ & $P$ \\
\hline Anthracycline only & 33 & & & & \\
\hline Taxane only & 25 & & 0.74 & $0.18-2.94$ & 0.66 \\
\hline Anthracycline plus taxane & 155 & 0.12 & 2.06 & $0.99-4.28$ & 0.05 \\
\hline$\leq 4$ & 59 & & & & \\
\hline $4-8$ & 110 & & & & \\
\hline$\geq 8$ & 45 & 0.42 & & & \\
\hline \multicolumn{6}{|l|}{ bPCR } \\
\hline \multicolumn{6}{|l|}{ aPCR } \\
\hline No & 145 & & & & \\
\hline Yes & 69 & 0.03 & 0.96 & $0.44-2.09$ & 0.92 \\
\hline \multicolumn{6}{|l|}{ sPCR } \\
\hline No & 99 & & & & \\
\hline Yes & 115 & $<0.01$ & 0.46 & $0.26-0.84$ & 0.01 \\
\hline
\end{tabular}

HR, hormone receptor; HER2, human epidermal growth factor receptor 2; AR, androgen receptor; CK5/6, cytokeratin; EGFR, epidermal growth factor receptor; PCR, pathologic complete response; sPCR, supraclavicular PCR; bPCR, breast PCR; aPCR, axillary PCR; -, negative; +, positive.

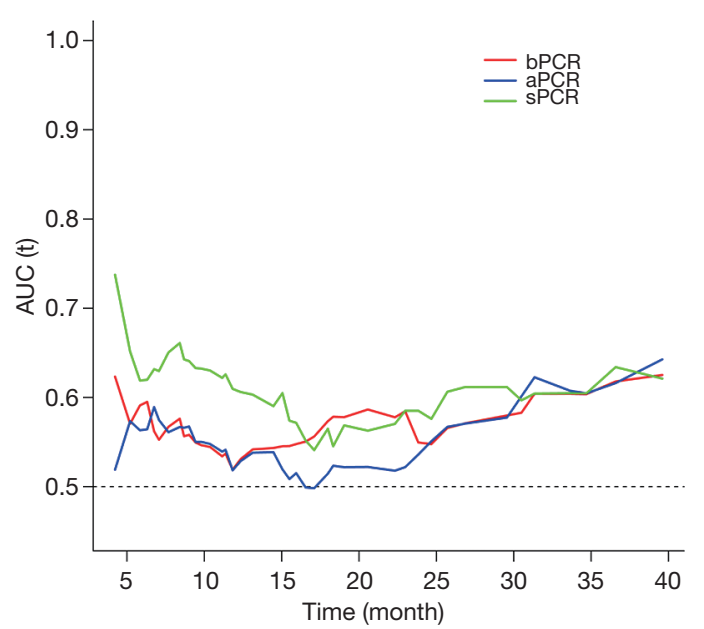

Figure 4 Evaluation of the prognostic value of sPCR, aPCR, and bPCR. Supraclavicular pCR performed better prognostic ability than the commonly used breast pCR and axillary pCR. studies (7), our work also found that ISLM was always found in patients with younger age, larger $T$ stage, HRnegative and HER2-positive tumors. It is suggested that patients with high risk factors of clinicopathological features may also be more likely to develop ISLNM at the time of initial diagnosis.

Based on the study by Brito et al. who put forward the idea that isolated ISLNM breast cancer was curable (8), patients with ISLNM was adjusted and included in the stage IIIc category of the tumor-nodemetastasis classification in the sixth edition of the AJCC staging in 2003. Therefore, patients with ISLNM but no other evidence of distant metastases warrant therapy administered with curative intent; i.e., combined-modality therapy consisting of neoadjuvant chemotherapy, surgery, radiotherapy, and endocrine therapy (9). However, there is still controversy pertaining to the treatment method of 
the local supraclavicular lymph node region in isolated ISLNM breast cancer. A host of researchers believe that supraclavicular area radiotherapy can benefit the patients in survival, which was also recommended in the National Comprehensive Cancer Network (NCCN) guidelines $(10,11)$. However, the value of supraclavicular lymph node dissection (SLND) in such patients is still under debate. On the one hand, a portion of studies assert that SLND can improve the survival of patients $(12,13)$, while others argued that these patients could not receive survival benefit from surgery (14). On the other hand, a plethora of studies have indicated that PCR in the breast and axillary sites should be proposed as a surrogate endpoint for the prediction of longterm clinical benefit after surgery as disease-free survival and overall survival, notably in patients with triple-negative and HER2-positive disease $(3,15)$. However, the related factors affecting supraclavicular pCR after NAC and its relationship between supraclavicular pCR and prognosis are not clear at present. Accordingly, there is growing scientific interest in the identification of predictive factors and the prognostic value of supraclavicular pCR.

NAC may change the treatment strategy of breast cancer by reducing the size and extent of primary tumors. For example, it was widely used to downstage locally advanced (inoperable) disease and make it operable, subsequently allowing for breast-conserving surgery (16). The research on the prediction model of breast pCR and axillary pCR has never stopped (17), but the research on supraclavicular pCR after NAC has not been reported. Our study found that the rate of supraclavicular pCR after NAC was $53.7 \%$, which was significantly higher than that of axillary pCR and breast pCR, suggesting that the metastasis in tumors of supraclavicular lymph nodes may be easier to be cleared through NAC. Multivariate analysis inferred that the expression levels of Ki67, axillary pCR, and breast pCR were significantly correlated with supraclavicular pCR. Furthermore, after the analysis of the 40 patients with high expression of Ki67 and having PCR both in the breast and axillary areas, we found that the rate of supraclavicular pCR was as high as $95 \%$, implying that the response of supraclavicular lymph nodes to NAC was consistent with that of primary breast lesions and axillary lymph nodes. Since the necessity of surgery in patients with breast pCR and axillary pCR after NAC also has been questioned $(18,19)$, and the rate of supraclavicular pCR can be accurately predicted by Ki67, breast pCR, and axillary pCR, it is open to question the necessity of supraclavicular surgery after NAC in such patients on the premise of preoperative identification of patients who have achieved breast pCR and axillary pCR (20). Inconsistent with previous studies which found that the pathologic response of breast and axillary were correlated with HR state and HER-2 state (21), our paper did not find this correlation with supraclavicular pCR, indicating that the response of supraclavicular lymph nodes to NAC is not completely consistent with that of breast and axillary sites perhaps because of the heterogeneity of tumors (22).

After a median follow-up of 16.2 months, Kaplan-Meier survival analysis showed that the risk of recurrence and metastasis in patients with supraclavicular pCR was 0.51 times higher than that in patients without supraclavicular pCR (HR 0.51, 95\% CI, 0.32-0.80, P<0.01). Cox regression analysis, adjusted for other prognostic factors, suggested a $54 \%$ reduction in the relative risk of relapse with supraclavicular pCR (HR 0.46, 95\% CI, 0.26-0.84, $\mathrm{P}=0.01$ ). Introducing the variable in our Cox multivariate model allowed us to verify that supraclavicular pCR was an independent predictor for DFS. Therefore, we can now state that supraclavicular pCR, similar to breast $\mathrm{pCR}$ and axillary pCR, can best discriminate between patients with favorable and unfavorable outcomes. Subgroup analysis also indicated that supraclavicular pCR was mainly manifested in HR-HER2- and HER2 positive disease. Notably, the predictive value for DFS was more pronounced in patients with supraclavicular pCR than in those with breast pCR (HR $0.48,95 \% \mathrm{CI}, 0.23-1, \mathrm{P}=0.05$ ) or axillary pCR (HR 0.96, $95 \% \mathrm{CI}, 0.44-2.09, \mathrm{P}=0.92)$. The prognostic value of breast pCR or axillary pCR may require a larger sample size to be statistically significant. Therefore, this research suggests that SLND in patients with ISLNM breast cancer can accurately obtain supraclavicular pCR after NAC, which can provide a more valuable reference for predicting the long-term outcomes of patients.

Our study has some strengths and limitations. This is so far, to our knowledge, the first and largest collective study on treated primary breast cancer with ISLNM which investigates supraclavicular pCR as a predictive and prognostic factor after NAC. The limitations are, first, that this is a retrospective report rather than a prospective clinical study with a higher level of evidence. Secondly, all patients collected in the study were treated with supraclavicular surgery combined with radiotherapy, and there were no patients who received radiotherapy alone. Finally, our early survival results need to be confirmed by a longer follow-up. The importance of a long-term follow-up is acknowledged, and these results, together with those of 
the overall survival analysis, will be reported in the future.

\section{Conclusions}

The findings from this study evaluating the largest group of patients with ISLNM at diagnosis indicate that ISLN was more likely to reach PCR than primary breast and axillary node metastases tumors after NAC. Supraclavicular PCR was highly correlated with the expression of Ki67, breast pCR, and axillary pCR. Our results also confirm that supraclavicular pCR was an independent predictor for DFS, mainly manifesting in HR-HER2 - and HER2positive diseases. This paper suggests that the utility of supraclavicular lymph node surgery in patients with ISLNM may be that it can accurately obtain supraclavicular pCR, which is of great significance for predicting the prognosis of patients.

\section{Acknowledgments}

We are grateful to Dr. Yizhou Jiang for his help with the preparation of the manuscript.

\section{Footnote}

Conflicts of Interest: The authors have no conflicts of interest to declare.

Ethical Statement: The study was carried out with the approval of the Ethical Review Committee of the Affiliated Cancer Hospital of Zhengzhou University. As this study was retrospectively designed, informed consent was waived by the Affiliated Cancer Hospital of Zhengzhou University. The authors are accountable for all aspects of the work in ensuring that questions related to the accuracy or integrity of any part of the work are appropriately investigated and resolved.

\section{References}

1. Debois JM. The significance of a supraclavicular node metastasis in patients with breast cancer. A literature review. Strahlenther Onkol 1997;173:1-12.

2. Singletary SE, Allred C, Ashley P, et al. Revision of the American Joint Committee on Cancer staging system for breast cancer. J Clin Oncol 2002;20:3628-36.

3. Berruti A, Amoroso V, Gallo F, et al. Pathologic complete response as a potential surrogate for the clinical outcome in patients with breast cancer after neoadjuvant therapy: a meta-regression of 29 randomized prospective studies. J Clin Oncol 2014;32:3883-91.

4. Goldhirsch A, Winer EP, Coates AS, et al. Personalizing the treatment of women with early breast cancer: highlights of the St Gallen International Expert Consensus on the Primary Therapy of Early Breast Cancer 2013. Ann Oncol 2013;24:2206-23.

5. Blumgart EI, Uren RF, Nielsen PM, et al. Predicting lymphatic drainage patterns and primary tumour location in patients with breast cancer. Breast Cancer Res Treat 2011;130:699-705.

6. Bonotto M, Gerratana L, Poletto E, et al. Measures of outcome in metastatic breast cancer: insights from a realworld scenario. Oncologist 2014;19:608-15.

7. Dellapasqua S, Bagnardi V, Balduzzi A, et al. Outcomes of patients with breast cancer who present with ipsilateral supraclavicular or internal mammary lymph node metastases. Clin Breast Cancer 2014;14:53-60.

8. Brito RA, Valero V, Buzdar AU, et al. Long-term results of combined-modality therapy for locally advanced breast cancer with ipsilateral supraclavicular metastases: The University of Texas M.D. Anderson Cancer Center experience. J Clin Oncol 2001;19:628-33.

9. Liu XH, Zhang L, Chen B. A meta-analysis of the prognosis in patients with breast cancer with ipsilateral supraclavicular lymph node metastasis versus patients with stage IIIb/c or IV breast cancer. Chronic Dis Transl Med 2015;1:236-42.

10. Fan Y, Xu B, Liao Y, et al. A retrospective study of metachronous and synchronous ipsilateral supraclavicular lymph node metastases in breast cancer patients. Breast 2010;19:365-9.

11. NCCN. NCCN Clinical Practice Guidelines in Oncology: Breast Cancer. version 3.2018. Available online: www.nccn. org 2018.

12. Zhang W, Qi XM, Chen AX, et al. The Role of Supraclavicular lymph node dissection in Breast Cancer Patients with Synchronous Ipsilateral Supraclavicular Lymph Node Metastasis. Zhonghua Zhong Liu Za Zhi 2017;39:374-9.

13. Jung J, Kim SS, Ahn SD, et al. Treatment Outcome of Breast Cancer with Pathologically Proven Synchronous Ipsilateral Supraclavicular Lymph Node Metastases. J Breast Cancer 2015;18:167-72.

14. Nikpayam M, Uzan C, Rivera S, et al. Impact of radical surgery on outcome in locally advanced breast cancer patients without metastasis at the time of diagnosis. 
Anticancer Res 2015;35:1729-34.

15. Zhang GC, Zhang YF, Xu FP, et al. Axillary lymph node status, adjusted for pathologic complete response in breast and axilla after neoadjuvant chemotherapy, predicts differential disease-free survival in breast cancer. Curr Oncol 2013;20:e180-92.

16. Killelea BK, Yang VQ, Mougalian S, et al. Neoadjuvant chemotherapy for breast cancer increases the rate of breast conservation: results from the National Cancer Database. J Am Coll Surg 2015;220:1063-9.

17. Ali HR, Dariush A, Thomas J, et al. Lymphocyte density determined by computational pathology validated as a predictor of response to neoadjuvant chemotherapy in breast cancer: secondary analysis of the ARTemis trial. Ann Oncol 2017;28:1832-5.

18. Tadros AB, Yang WT, Krishnamurthy S, et al. Identification of Patients With Documented Pathologic Complete Response in the Breast After Neoadjuvant Chemotherapy for Omission of Axillary Surgery. JAMA

Cite this article as: Zhu J, Jiao D, Guo X, Qiao J, Ma Y, Zhang J, Chen H, Xiao H, Yang Y, Lu Z, Liu Z. Predictive factors and prognostic value of pathologic complete response of ipsilateral supraclavicular lymph nodes in breast cancer after neoadjuvant chemotherapy. Ann Transl Med 2019;7(22):666. doi: 10.21037/ atm.2019.10.22
Surg 2017;152:665-70.

19. Barron AU, Hoskin TL, Day CN, et al. Association of Low Nodal Positivity Rate Among Patients With ERBB2-Positive or Triple-Negative Breast Cancer and Breast Pathologic Complete Response to Neoadjuvant Chemotherapy. JAMA Surg 2018;153:1120-6.

20. Kuerer HM, Rauch GM, Krishnamurthy S, et al. A Clinical Feasibility Trial for Identification of Exceptional Responders in Whom Breast Cancer Surgery Can Be Eliminated Following Neoadjuvant Systemic Therapy. Ann Surg 2018;267:946-51.

21. von Minckwitz G, Untch M, Blohmer JU, et al. Definition and impact of pathologic complete response on prognosis after neoadjuvant chemotherapy in various intrinsic breast cancer subtypes. J Clin Oncol 2012;30:1796-804.

22. Zardavas D, Irrthum A, Swanton C, et al. Clinical management of breast cancer heterogeneity. Nat Rev Clin Oncol 2015;12:381-94. 